\title{
Estudo do óleo essencial de algumas espécies do gênero Baccharis (Asteraceae) do sul do Brasil
}

\author{
F. Agostini ${ }^{1 *}$, A.C.A. Santos ${ }^{1,2}$, M. Rossato ${ }^{1,3}$, M.R. Pansera ${ }^{1}$, F. Zattera ${ }^{1}$, R. Wasum ${ }^{4}$, \\ L.A. Serafini, ${ }^{1,2}$ \\ ${ }^{1}$ Instituto de Biotecnologia, Universidade de Caxias do Sul, Rua Francisco Getúlio Vargas, 1130, Petrópolis, \\ 95070-560, Caxias do Sul, RS, Brasil, \\ ${ }^{2}$ Departamento de Física e Química, Universidade de Caxias do Sul, Rua Francisco Getúlio Vargas, 1130, \\ Petrópolis, 95070-560, Caxias do Sul, RS, Brasil, \\ ${ }^{3}$ Departamento de Ciências Exatas e da Natureza, Universidade de Caxias do Sul, Campus da Região dos vinhedos, \\ Alameda João Dal Sasso, 800, 95700-000, Bento Gonçalves, RS, Brasil, \\ ${ }^{4}$ Herbário, Universidade de Caxias do Sul, Rua Francisco Getúlio Vargas, 1130, Petrópolis, \\ 95070-560, Caxias do Sul, RS, Brasil
}

\begin{abstract}
RESUMO: Cerca de 15\% das espécies de Baccharis têm sido analisadas do ponto de vista fitoquímico, mas trabalhos envolvendo estudos de óleos essenciais têm sido pouco informados. Este trabalho teve como objetivo identificar espécies deste gênero, nativas do Rio Grande do Sul, e avaliar o potencial aromático de doze amostras coletadas em diferentes localidades do Estado. O óleo foi obtido por hidrodestilação em Clevenger por 1 hora a partir do material desidratado a $30^{\circ} \mathrm{C}$ por quatro dias. As análises em CG foram realizadas em equipamento HP6890 Series, equipado com processador de dados HP-Chemstation e coluna HP-Innowax, e, as análises em CG/EM foram realizadas em equipamento HP 6890/MSD5973, com software HP Chemstation e biblioteca Wiley 275 de espectros, com coluna de mesma polaridade. O melhor rendimento foi obtido em $B$. articulata com 0,5\% p/v. $\beta$-Pineno foi identificado nas amostras de B. articulata (41,4\% a 52,8\%), e em B. cogata (27,2\%). Espatulenol esteve presente nas amostras de B. semiserrata (15,5\% e 25,5\%) e nas amostras de $B$. uncinella $(47,7 \%$ e $23,1 \%)$. B. oxyodonta apresentou limoneno $(24,3 \%)$. Duas amostras de $B$. milleflora apresentaram espatulenol, enquanto que uma apresentou $\beta$-pineno.
\end{abstract}

Unitermos: Baccharis, plantas nativas, óleo essencial, hidrodestilação.

\begin{abstract}
Studies on the essential oils from several Baccharis (Asteraceae) from Southern Brazil". About 15\% of the known Baccharis species have been phytochemically studied, but few studies regarding their essential oils have been reported. The present work aims to identify native species of the State of Rio Grande do Sul, and to evaluate their aromatic potential. Twelve samples were collected in different sites within the State. The essential oils were obtained by an one hour hydrodistillation in a Clevenger apparatus, using plant materials dried for four days at $30^{\circ} \mathrm{C}$. The GC analyses were carried out with a HP6890 Series apparatus, equiped with a HP-Chemstation data processor and an HP-Innowax column. The GC/MS analyses were carried out with an HP 6890/MSD5973, equiped with an HP Chemstation and a Wiley 275 spectra library, using the same column as above. The best yield was those of $B$. articulata $(0.5 \% \mathrm{v} / \mathrm{w}) . \beta$-Pinene was identified as the main component in the samples from B. articulata (41.4\% to $52.8 \%$ ) and from B. cogata (27.2\%). Spathulenol was present in the samples from B. semiserrata (15.5\% and $25.5 \%)$ and those from B. uncinella (47.7\% and 23.1\%). B. oxyodonta showed limonene (24.3\%). Two samples of $B$. milleflora had spathulenol as main component, but a third one had $\beta$-pinene as main constituent.
\end{abstract}

Keywords: Baccharis, native plants, essential oils, hydrodistillation.

\section{INTRODUÇÃO}

Plantas do gênero Baccharis, conhecidas popularmente como carquejas, são arbustos lenhosos de grande diversidade morfológica pertencentes a família Asteraceae, uma família que possui cerca de 1.100 gêneros e 25.000 espécies distribuídas por todo o mundo (Moreira et al., 2003). Várias plantas desta família são produtoras de óleo essencial de importância comercial, principalmente na Europa e nos Estados Unidos, sendo usados, em sua maioria, nas indústrias de perfumes e licores (Craveiro et al., 1981).

O gênero Baccharis está representado por mais de 500 espécies distribuídas principalmente no Brasil, Argentina, Colômbia, Chile e México (Verdi, et al, 2005). Nesta vasta área se encontra profundamente diversificado, ocupando grande variedade de ambientes e constituíndo um importante elemento em numerosas formações vegetativas (Giuliano, 2001). No Brasil, estão descritas 120 espécies de Baccharis, distribuídas em maior concentração na Região Sul do país (Barroso, 1976; Verdi, et al, 2005). As espécies deste gênero são subarbustos ou 
arbustos ramificados, com 0,5 a 4 metros de altura (Boldt, 1989), com caule e ramos cilíndricos, folhas alternas e muito variáveis na forma e no tamanho, e com capítulos que podem ser de uni a multiflores (Barroso, 1976; Joly, 1993). São plantas dióicas com inflorescências masculinas e femininas em plantas separadas (Boldt, 1989; Ferracini et al., 1995).

As espécies pertencentes a este gênero apresentam elevado valor sócio-econômico, com ampla dispersão nos estados de Santa Catarina, Paraná, São Paulo e Rio Grande do Sul, entre outras regiões do país, onde grande número delas são utilizadas na medicina popular para controle ou tratamento de várias doenças. São consumidas principalmente na forma de chás com indicações para males do estômago, fígado, anemias, inflamações, diabetes, doenças na próstata, sendo também descritas como remédio para o processo de desintoxicação do organismo (Verdi, et al, 2005).

Este gênero produz muitos metabólitos secundários, sendo que cerca de $15 \%$ das espécies de Baccharis têm sido analisadas do ponto de vista fitoquímico, mas poucas destas espécies têm estudos completos (Boldt, 1989). Cerca de 120 espécies deste gênero foram estudadas quimicamente e de modo geral, os compostos que mais se destacam são os flavonóides e os terpenóides (Verdi, et al, 2005; Davies, 2004) como monotepenos, sesquitepenos, diterpenos e triterpenos (Moreira, et al, 2003; Davies, 2004). Muitos terpenóides ocorrem como componentes de folhas e ramos de espécies deste gênero (Boldt, 1989), podendo ser citados nerolidol, espatulenol, $\delta$-cadineno e $\beta$-cariofileno encontrados em Baccharis dracunculifolia (Queiroga et al., 1990). Outras espécies que apresentam terpenóides são Baccharis salicifolia, com $\alpha$-felandreno, germacreno-D, biciclogermacreno e $\delta$-cadineno entre outros, e Baccharis latifolia, apresentando $\alpha$-tujeno, $\alpha$-pineno, limoneno, germacreno-D e ledol como terpenóides majoritários (Loaysa et al., 1995).

Os óleos essenciais extraídos de folhas de Baccharis dracunculifolia (óleo-de-vassoura) e Baccharis trimera (óleo-de-carqueja) são produzidos e usados em perfumaria, possuindo alto valor para indústrias de fragrâncias (Boldt, 1989; Ferracini et al., 1995; Verdi, et al, 2005).

\section{MATERIAL E MÉTODOS}

\section{Coleta do material vegetal}

Foram coletadas doze amostras pertencentes a seis espécies de Baccharis, em diferentes localidades do Rio Grande do Sul. Na Tabela 1 podem ser observados os dados de coleta e de herbário referentes às espécies estudadas. A classificação das plantas foi realizada pelo Dr. Ronaldo Wasum, Botânico do Herbário da Universidade de Caxias do Sul.

\section{Preparação do material vegetal}

Para realização das extrações de óleos essenciais foram coletados aproximadamente $500 \mathrm{~g}$ do material fresco, o qual foi encaminhado à sala de secagem, com temperatura controlada de $30^{\circ} \mathrm{C}$ e circulação de ar forçada, pelo período de aproximadamente 4 dias, para desidratação.

\section{Preparação das exsicatas}

Foram coletadas aproximadamente 4 a 6 exemplares de cada amostra, sendo estes, ramos contendo flores e folhas adultas. Este material foi fichado, herborizado e encaminhado ao Herbário de Universidade de Caxias do Sul (3 exemplares) para identificação taxonômica, sendo que os demais exemplares foram permutados com Herbários Nacionais e Internacionais.

\section{Extração do óleo essencial}

O óleo essencial foi obtido da parte aérea das plantas por hidrodestilação em aparelho Clevenger (Mechkovski; Akerele, 1992) pelo período de 1 hora, utilizando-se cerca de 100 gramas de planta seca. O

Tabela 1. Descrição das espécies de Baccharis estudadas.

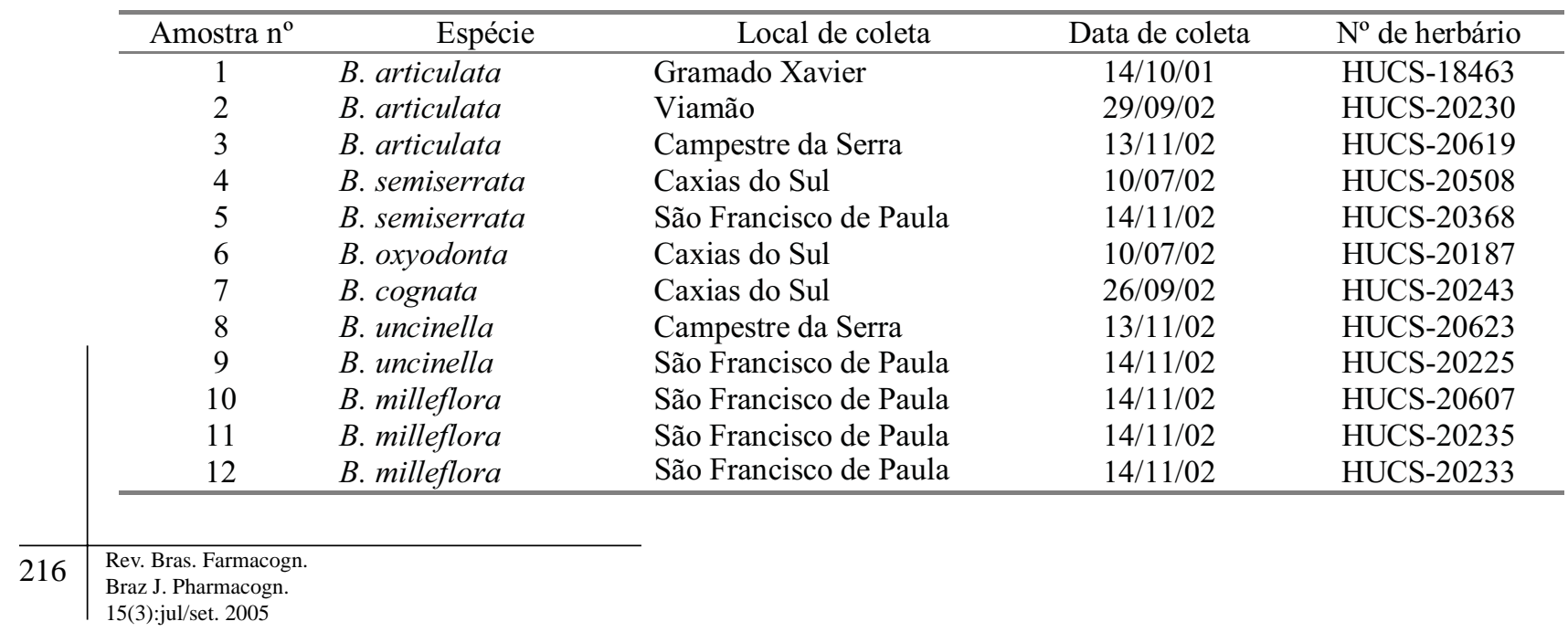


Tabela 2. Composição química de B. articulata, B. semisserrata, B. oxyodonta B.cognata, B. uncinella e B. milleflora

\begin{tabular}{|c|c|c|c|c|c|c|c|c|c|c|c|c|c|c|}
\hline \multirow[t]{2}{*}{ Composto } & \multirow{2}{*}{ R.T. } & \multicolumn{12}{|c|}{ Área (\%) } & \multirow[t]{2}{*}{ R.I. } \\
\hline & & 1 & 2 & 3 & 4 & 5 & 6 & 7 & 8 & 9 & 10 & 11 & 12 & \\
\hline $\begin{array}{l}\text { Rendimento de óleo } \\
(\% \mathrm{v} / \mathrm{p})\end{array}$ & - & 0,5 & 0,2 & 0,2 & 0,1 & 0,1 & 0,3 & 0,1 & 0,2 & 0,2 & 0,1 & 0,1 & 0,1 & - \\
\hline$\alpha$-Pineno & 4,34 & 2,1 & 7,0 & 2,6 & 7,8 & 1,4 & - & 5,7 & 8,1 & 12,9 & - & 0,3 & 2,9 & 1011 \\
\hline$\alpha$-Tujeno & 4,98 & - & - & - & - & - & - & 1,2 & 2,4 & 2,4 & - & - & - & 1017 \\
\hline$\beta$-Pineno & 7,63 & 41,4 & 52,8 & 46,5 & 5,8 & 6,8 & 9,3 & 27,2 & 10,2 & 9,8 & - & 4,8 & 34,2 & 1070 \\
\hline Sabineno & 9,13 & - & 1,1 & - & 0,4 & 0,3 & 20,5 & 1,8 & - & 1,1 & - & - & - & 1080 \\
\hline Mirceno & 11,84 & - & 1,5 & - & 2,6 & 2,7 & 5,0 & 1,4 & - & 1,3 & - & - & 0,1 & 1150 \\
\hline$\alpha$-Terpineno & 11,95 & - & - & - & - & - & - & - & - & 0,6 & - & - & - & 1171 \\
\hline Limoneno & 12,70 & 17,6 & 4,0 & 16,1 & 11,6 & 3,3 & 24,3 & 10,3 & 16,8 & 14,2 & - & 1,8 & 2,4 & 1201 \\
\hline$\beta$-Felandreno & 12,96 & - & - & - & - & - & 6,4 & - & - & - & - & - & - & 1206 \\
\hline cis-Ocimeno & 13,32 & - & - & - & - & - & - & - & - & - & 1,4 & - & - & 1220 \\
\hline trans- $\beta$-Ocimeno & 15,71 & - & - & - & - & - & - & - & - & - & 1,5 & - & - & 1243 \\
\hline$\alpha$-Terpinoleno & 15,68 & - & - & - & - & - & 1,5 & - & - & - & - & - & - & 1268 \\
\hline$p$-Cimeno & 17,01 & - & 0,6 & - & - & - & 0,7 & - & - & 0,5 & - & - & - & 1272 \\
\hline cis-Sabineno hidrato & 25,35 & - & - & - & - & - & 0,2 & - & - & - & - & - & - & 1443 \\
\hline$\alpha$-Gurjuneno & 27,49 & - & - & - & - & - & 0,3 & - & - & - & 1,2 & - & - & 1469 \\
\hline$\alpha$-Copaeno & 30,06 & - & - & - & 2,5 & - & - & - & - & - & - & 0,2 & - & 1485 \\
\hline$\beta$-Cariofileno & 30,49 & - & - & 1,5 & 6,7 & - & 1,6 & 2,7 & 1,6 & 1,5 & 7,1 & 1,9 & 0,9 & 1547 \\
\hline Terpinen-4-ol & 31,27 & - & - & - & 7,4 & - & 1,2 & - & - & 2,1 & - & 0,1 & - & 1553 \\
\hline$\beta$-Selineno & 33,40 & - & - & - & - & - & - & - & - & 0,3 & 3,2 & - & - & 1558 \\
\hline Germacreno-D & 34,83 & - & 1,8 & 2,9 & 0,6 & 1,3 & 2,8 & 1,1 & 0,9 & 2,1 & 3,4 & 0,4 & 2,0 & 1599 \\
\hline Biciclogermacreno & 35,74 & - & 0,7 & 0,9 & - & 1,7 & 3,5 & 2,6 & 1,6 & 1,1 & - & 0,7 & 0,6 & 1652 \\
\hline$\alpha$-Muuroleno & 35,86 & - & - & - & - & - & - & - & - & - & - & - & 0,6 & 1655 \\
\hline$\delta$-Cadineno & 36,77 & - & - & 0,7 & - & - & - & - & 0,5 & 0,6 & - & 2,7 & 4,9 & 1656 \\
\hline Mirtenol & 38,11 & - & - & - & - & - & - & 0,7 & - & - & - & 0,3 & 1,3 & 1689 \\
\hline Germacreno-A & 38,43 & - & - & - & 1,2 & 0,3 & - & - & - & - & - & - & - & 1691 \\
\hline Ar-curcumeno & 39,62 & - & - & - & 0,1 & - & - & - & - & - & - & - & - & 1768 \\
\hline Palustrol & 42,34 & - & - & - & - & - & - & - & - & - & - & 1,1 & 1,2 & 1817 \\
\hline Óxido de cariofileno & 43,88 & - & 1,9 & 1,9 & - & - & - & 5,9 & 8,2 & 4,5 & - & 8,9 & 3,8 & 1870 \\
\hline Metil eugenol & 45,47 & - & - & 0,7 & 1,0 & 0,9 & - & - & - & - & - & - & - & 1965 \\
\hline Aromadendreno & 46,90 & - & - & - & 0,8 & 0,4 & - & - & - & - & - & 0,4 & 1,6 & 1972 \\
\hline Globulol & 47,02 & - & - & - & - & - & - & - & - & - & - & 0,9 & - & 1973 \\
\hline Viridiflorol & 47,23 & - & 4,4 & - & - & - & - & - & - & - & - & 1,3 & - & 1975 \\
\hline$\gamma$-Gurjuneno & 47,24 & - & - & - & - & - & - & - & - & - & 0,2 & - & - & 1977 \\
\hline Ledeno & 47,28 & - & - & - & - & - & - & - & - & 1,6 & 2,2 & - & - & 1978 \\
\hline Elemol & 47,45 & - & - & - & 0,9 & 5,7 & - & - & - & - & - & - & - & 1994 \\
\hline Espatulenol & 48,25 & 1,4 & 10,4 & 7,4 & 15,5 & 25,5 & 6,7 & 20,7 & 47,7 & 23,1 & 25,3 & 16,2 & 10,7 & 2025 \\
\hline$\beta$-Eudesmol & 50,85 & - & - & - & 0,9 & 0,4 & - & - & - & - & - & - & - & 2199 \\
\hline $\begin{array}{l}\text { Monoterpenos } \\
\text { hidrocarbonados }\end{array}$ & - & 61,1 & 67,0 & 62,3 & 28,2 & 14,6 & 68,0 & 47,5 & 37,4 & 42,7 & 2,9 & 6,3 & 38,4 & - \\
\hline $\begin{array}{l}\text { Sesquiterpenos } \\
\text { hidrocarbonados }\end{array}$ & - & - & 2,6 & 5,9 & 11,9 & 3,7 & 8,2 & 6,5 & 4,9 & 7,2 & 14,4 & 6,8 & 12,4 & - \\
\hline $\begin{array}{l}\text { Monoterpenos } \\
\text { oxigenados }\end{array}$ & - & - & - & 0,7 & 8,4 & 0,9 & 1,2 & - & - & 2,1 & - & 0,1 & - & - \\
\hline $\begin{array}{l}\text { Sesquiterpenos } \\
\text { oxigenados }\end{array}$ & - & 1,4 & 16,7 & 9,3 & 17,3 & 31,6 & 6,7 & 27,2 & 55,9 & 27,6 & 25,3 & 28,2 & 17,1 & - \\
\hline $\begin{array}{l}\text { Total de compostos } \\
\text { identificados }\end{array}$ & - & 62,4 & 86,3 & 78,2 & 65,9 & 50,8 & 84,1 & 81,2 & 98,2 & 79,7 & 45,6 & 42,1 & 67,9 & - \\
\hline
\end{tabular}

Espécies: 1 , 2 e $3=$ B. articulata; 4 e $5=$ B. semisserrata; $6=$ B. oxyodonta; $7=$ B. cognata; 8 e $9=B$. uncinella; 10,11 e $12=B$. milleflora

R.T. Tempo de Retenção do composto

R.I. Índice de Retenção de Kovats

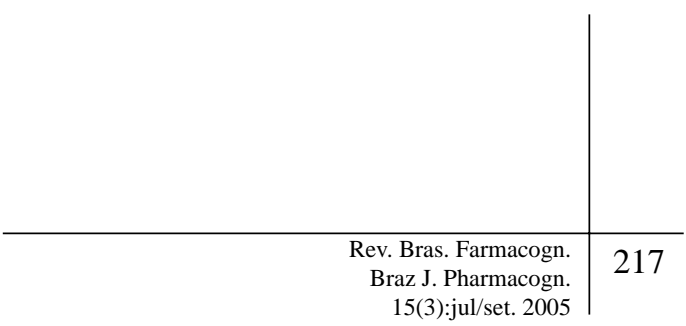


rendimento de óleo essencial em $\mathrm{mL}$ foi lido diretamente na bureta graduada do aparelho Clevenger.

\section{Análise do óleo essencial}

A identificação dos componentes químicos das amostras de óleo essencial foi feita por Cromatografia Gasosa (CG) e Cromatografia Gasosa acoplada a Detector Seletivo de Massas (CG/EM).

Os constituintes dos óleos foram identificados por comparação de seus espectros de massas com aqueles da biblioteca Wiley (GC/MS) e com aqueles descritos por Adams (1995), bem como, por comparação do índice de retenção de Kovats com dados da literatura (Adams, 1995). O índice de Kovats foi calculado através da equação de Kovats, utilizando-se uma solução padrão de hidrocarbonetos $\mathrm{C}_{9}$ a $\mathrm{C}_{25}$.

As análises em CG foram realizadas num cromatógrafo Hewlett Packard 6890 Series, equipado com um processador de dados HP-Chemstation, utilizando-se uma coluna HP-Innowax (30 m x $320 \mu \mathrm{m}$ i.d.) 0,50 $\mu \mathrm{m}$ espessura de filme (Hewlett Packaard, Palo Alto, USA), temperatura da coluna, $40^{\circ} \mathrm{C}$ (8 min) para $180^{\circ} \mathrm{C}$ a $3^{\circ} \mathrm{C} / \mathrm{min}, 180-230^{\circ} \mathrm{C}$ a $20^{\circ} \mathrm{C} / \mathrm{min}, 230^{\circ} \mathrm{C}(20$ min); temperatura de injetor $250^{\circ} \mathrm{C}$; razão de split $1: 50$, detector de ionização de chama com temperatura de $250^{\circ} \mathrm{C}$; gás de arraste $\mathrm{H}_{2}$ (34Kpa), volume injetado $1 \mu \mathrm{L}$ diluído em hexano (1:10).

As análises em CG/EM foram realizadas num cromatógrafo gasoso acoplado a detector seletivo de massas Hewlett Packard 6890/MSD5973, equipado com software HP Chemstation e biblioteca Wiley 275 de espectros. Foi utilizada coluna HP-Innowax (30 m x $250 \mu \mathrm{m}$ ) 0,50 $\mu \mathrm{m}$ espessura de filme (Hewlett Packard, Palo Alto, USA). O programa de temperatura utilizado foi o mesmo usado no CG: interface $280^{\circ} \mathrm{C}$; razão de split 1:100; gás de arraste He (56 Kpa); razão de fluxo: 1,0 $\mathrm{mL} / \mathrm{min}$; energia de ionização $70 \mathrm{eV}$; volume injetado 0,6 $\mu \mathrm{L}$ diluído em hexano (1:10).

\section{RESULTADOS E DISCUSSÃO}

Os rendimentos dos óleos essenciais estudados variaram de $0,1 \% \mathrm{p} / \mathrm{v}$ a $0,5 \% \mathrm{p} / \mathrm{v}$, sendo que os maiores rendimentos foram observados para $B$. articulata com $0,5 \% \mathrm{p} / \mathrm{v}$ e $B$. oxyodonta com $0,3 \% \mathrm{p} / \mathrm{v}$ (Tabela 2 ).

As análises cromatográficas das doze amostras pertencentes a seis diferentes espécies de Baccharis também podem ser observadas na Tabela 2 .

As três amostras de $B$. articulata apresentaram variações quali-quantitativas na composição química, relacionadas à concentração de $\alpha$-pineno, limoneno e espatulenol; por outro lado, $\beta$-pineno foi o composto majoritário encontrado nas três amostras. Apresentaram também variações no rendimento. É provável que estas diferenças sejam devidas ao estádio de desenvolvimento da planta e a condições ambientais e geográficas em geral, já que as amostras foram coletadas em diferentes localidades do Rio Grande do Sul.

B. semiserrata apresentou rendimento de $0,1 \%$ $\mathrm{p} / \mathrm{v}$ e como composto químico majoritário o espatulenol em ambas as amostras estudadas. As diferenças observadas na composição química minoritária, tanto quantitativas quanto qualitativas, são provavelmente devidas a diferenças geográficas e, principalmente, à época de coleta, pois a amostra 4 foi coletada no inverno e a amostra 5, na primavera.

As amostras de B. milleflora estudadas apresentaram compostos químicos majoritários distintos, provavelmente pela presença de quimiotipos nas mesmas, visto que foram coletadas em mesmo local e época. Por outro lado, apresentaram mesmo rendimento percentual nas três amostras estudadas, provavelmente pelo mesmo motivo. Estes dados indicam a necessidade de um estudo mais detalhado em relação a esta espécie.

As duas amostras de $B$. uncinella apresentaram como composto majoritário, o espatulenol seguido de limoneno, e ainda, rendimento de $0,2 \% \mathrm{p} / \mathrm{v}$ para ambas as espécies.

B. cognata apresentou como composto majoritário $\beta$-pineno e $B$. oxyodonta, limoneno.

Entre todas as amostras, algumas podem ser destacadas por apresentarem compostos em concentrações elevadas, tais como a amostra 2, B. articulata, com 52,8\% de $\beta$-pineno, a amostra $6, B$. oxyodonta, apresentou limoneno, 24,3\%, e a amostra 5, B. semiserrata, espatulenol, 25,5\%.

Segundo Price; Price (1999) e Simões; Spitzer (2000), numa determinada espécie, o rendimento de óleo essencial e a concentração de cada um dos constituíntes deste óleo pode variar durante o desenvolvimento do vegetal, o ambiente no qual ovegetal se desenvolvetambém influencia na composição química dos óleos essenciais e no seu rendimento, a temperatura, a umidade relativa, a duração total de exposição ao sol e o regime de ventos exercem influência direta na composição e rendimento, e ainda, diferentes órgãos de uma mesma planta podem apresentar óleos essenciais com composição química, caracteres físico-químicos e odores bem distintos.

\section{CONCLUSÕES}

O melhor rendimento de óleo essencial foi obtido em $B$. articulata com $0,5 \% \mathrm{p} / \mathrm{v}$.

As três amostras de $B$. articulata estudadas apresentaram como composto majoritário o $\beta$-pineno, em concentrações que variaram de $41,4 \%$ a 52,8\%, além de $B$. cogata, com $27,2 \%$ deste mesmo composto. As duas amostras de $B$. semisserrata apresentaram $15,5 \%$ e $25,5 \%$ de espatulenol, e as duas amostras de $B$. uncinella apresentaram $47,7 \%$ e $23,1 \%$ deste composto. B. oxyodonta apresentou $24,3 \%$ de limoneno. 
Duas amostras de B. milleflora apresentaram como composto majoritário espatulenol, enquanto que uma amostra apresentou $\beta$-pineno como majoritário, provavelmente pela presença de quimiotipos nesta espécie, o que poderá ser comprovado com estudos mais detalhados.

\section{AGRADECIMENTOS}

À Coordenação de Aperfeiçoamento de Pessoal de Nível Superior / Conselho Nacional de Pesquisa (CAPES/CNPq), Universidade de Caxias do Sul (UCS) e Secretaria de Ciência e Tecnologia do Estado do Rio Grande do Sul (SCT-RS).

\section{REFERÊNCIAS}

Adams RP 1995. Identification of essential oils by ion trap mass spectroscopy. California: Academic Press, Inc.

Barroso GM 1976. Compositae - Subtribo Baccharidinae Hoffmann: Estudo das espécies ocorrentes no Brasil. Rodriguésia 40: 28-31.

Boldt PE 1989. Baccharis (Asteraceae), a review of its taxonomy, phytochemistry, ecology, economic status, natural enemies and the potential for its biological control in the United States. Texas: College Station.

Craveiro AA, Fernandes AG, Andrade CHS, Matos FJA, Alencar JWD, Machado MIL 1981. Óleos essenciais de plantas do nordeste. Fortaleza: UFC.

Davies P 2004. Fichas técnicas de cultivo. In: Estudios en domesticación y cultivo de especies medicinales y aromáticas nativas. Publicação Técnica do Instituto Nacional de Investigacion Agropecuaria (INIA), Projeto FPTA 137, Uruguai.

Ferracini VL, Paraiba LC, Leitão Filho HF, Silva AGD, Nascimento LR, Marsaioli AJ 1995. Essential oils of seven Brazilian Baccharis species. J Essent Oil Res 7: 355-367.

Giuliano DA 2001. Clasificación infragenérica de las espécies Argentinas de Baccharis (Asteraceae, Astereae). Darwiniana 39: 131-154.

Joly AB 1993. Botânica: Introdução à taxonomia vegetal. $11^{a}$ ed. São Paulo: Companhia Editora Nacional.

Loaysa I, Abujder D, Aranda R, Jakupovic J, Collin G, Deslauriers H, Jean FI 1995. Essential oils of Baccharis salicifolia, B. latifolia and B. dracunculifolia. Phytochemistry 38: 381-389.

Mechkovski A, Akerele CO 1992. Quality control methods for medicinal plant materials. Who/Pharm/92.559. Switzerland: World Health Organization.

Moreira FPM, Coutinho V, Montanher ABP, Caro MSB, Brighente IMC, Pizzolatti MG 2003. Flavonóides e triterpenos de Baccharis pseudotenuifolia Bioatividade sobre Artemisia salina. Quím. Nova 26: 309-311.

Price S, Price L 1999. Aromatherapy for health professionals. $2^{\text {nd }}$ ed. New York: Churchill Livingstone.

Queiroga CL, Fukai A, Marsaioli A 1990. Composition of the essential oil of vassoura. J Braz Chem Soc 1: 105109.

Simões CMO, Spitzer V 2000. Óleos voláteis. In: Simões
CMO, Schenkel EP, Gosmann G, Mello JCPD, Mentz LA, Petrovick PR. Farmacognosia da planta ao medicamento. Porto Alegre: Editora da Universidade Federal do Rio Grande do Sul, p. 387-415.

Verdi LG, Brighente IMC, Pizzolatti MG 2005. Gênero Baccharis (Asteraceae): Aspéctos químicos, econômicos e biológicos. Quím. Nova 28: 85-94.

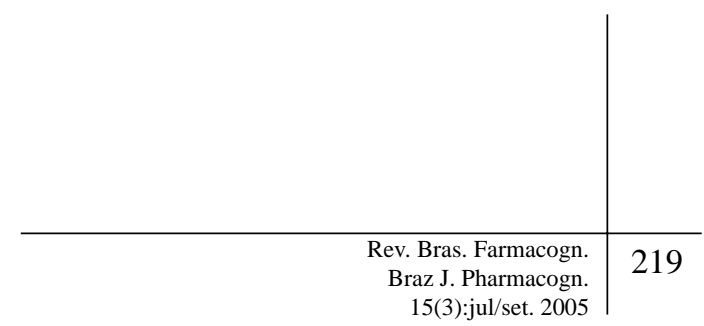

